\title{
Calculation of the effect of plastic deformation on thermo-kinetic electromotive force in TiNi alloy
}

\author{
A. V. Lesota ${ }^{1, \dagger}$, V. V. Rubanik ${ }^{1,2}$, V.V. Rubanik Jr. ${ }^{1}$ \\ †ann20zv@tut.by \\ ${ }^{1}$ Institute of Technical Acoustics of NAS of Belarus, 13 General Lyudnikov Ave., Vitebsk, 210009, Belarus \\ ${ }^{2}$ Vitebsk State Technological University, 72 Moskovskiy Ave., Vitebsk, 210035, Belarus
}

\begin{abstract}
It is known that the thermo-kinetic electromotive force (EMF) in TiNi is induced due to thermoelastic phase transformations. The occurrence of the thermo-kinetic EMF is possible with the movement of both the local heating and cooling zones along the extended TiNi sample. Local heating of the conductor determines the reverse phase transformation $\left(T \geq A_{s}\right)$, while cooling determines the forward one $\left(T \leq M_{s}\right)$. When the thermo-kinetic EMF is induced in a TiNi wire sample, two successive EMF peaks opposite in sign are observed when the cooling zone moves through the deformation zone $\left(L_{\text {def }}<1.4 \mathrm{~cm}\right)$; after the cooling zone passes through the deformation zone, the thermo-kinetic EMF parameter returns to its initial value. This paper presents a physical model according to which the change in the thermo-kinetic EMF value when the cooling zone moves through the deformation zone is associated with the appearance of the potential difference at the boundaries of the deformation zone due to changes in temperature and differences between the thermo-EMF coefficient values on the plastically deformed (due to the accumulated defects) and undeformed zones of the TiNi wire sample. Mathematical expressions were obtained to calculate the change of the thermo-kinetic EMF value during the movement of the cooling zone through the zone of deformation in TiNi alloy, as well as the dependence of this value on the deformation degree, the length of the deform zone, and the temperature in the cooling zone. The calculation results are in good agreement with the experimental data and can be used to develop methods and tools for homogeneity control of TiNi products.
\end{abstract}

Keywords: TiNi alloys, thermo-kinetic EMF, forward phase transformation, plastic deformation.

\section{Introduction}

Unique properties of titanium nickelide find application in engineering practice (operating mechanisms and power equipment), medical industry (to manufacture implantable devices and tools capable of changing the shape of the working part), robotics, etc [1,2,3]. Design of new products with the shape memory effect (SME) imposes definite requirements on the uniformity of physico-mechanical and functional properties of the materials, i.e. there is necessity to create methods, techniques, and quality control devices for extended TiNi products.

Electronic properties of metals such as resistance, heat conductivity, thermal electromotive force (EMF) coefficient are particularly sensitive to deformation of metals. Moreover, the change of electronic properties of metals caused by plastic deformation results in changes in thermoelectric phenomena observed in the materials under consideration $[4,5,6]$. The thermo-kinetic EMF occurring in homogeneous materials and first observed when moving a local heating zone along an iron wire sample or tungsten film is among the phenomena $[7,8]$; in this case the mandatory condition for such EMF to appear is a phase transformation in the local heating zone. In materials with the EMF phase transformations proceed at low temperatures (down to negative ones). During thermoelastic phase transformations the EMF initiation is possible when moving both the heating zone $[9,10,11]$ and the local cooling zone [12] along the conductor. It also is known that the value of the EMF induced increases when moving the cooling zone through the local deformation zone with the EMF increase depending on the TiNi sample deformation degree [13]. It should be noted that physical nature of the phenomenon is still not fully understood. The purpose of the study was to investigate the impact of plastic deformation of extended TiNi products on the value of the thermo-kinetic EMF induced in them.

\section{Computational methodology}

It is known [14] that in the process of movement of the cooling zone $\left(T<M_{f}\right)$ at constant velocity along the extended TiNi sample (in the austenitic phase state) phase transformations occur on both sides of the cooling zone. Cooling results in forward phase transformation at the leading edge, while heating the cooled zone by means of heat exchange with the environment results in reverse phase transformation at the trailing edge.

The authors assume that contact potential difference caused by the interaction of austenitic and martensitic phases with different values of electronic density of states at Fermi level [15] occurs in the zones of phase transformations. Therefore, when moving the cooling zone along the TiNi wire sample, the thermo-EMF occurs in the regions with forward and reverse phase transformations due to the contact potential difference between austenite and martensite. The values of the thermo-EMF in the regions with forward and reverse phase 
transformations are different due to different temperatures of martensitic transitions of the transformations. Therefore, the value of the thermo-kinetic EMF occurring as a result of the movement of the cooling zone $\left(T<M_{f}\right)$ along the TiNi sample equals the difference in values of the thermo-EMF in the regions with forward and reverse phase transformations.

To calculate the thermo-kinetic EMF value $(E)$ the following formula is suggested [14]:

$$
\begin{aligned}
& E\left(t, T_{\min }\right)=\int_{x=0}^{x=x_{0}+v t}\left[\left(\frac{\partial S_{f}(T(x, t))}{\partial x}\right) \cdot\left(T(x, t)-T^{0}\right)\right] d x- \\
& -\int_{x=x_{0}+v t}^{x}\left[\left(\frac{\partial S_{r}(T(x, t))}{\partial x}\right) \cdot\left(T(x, t)-T^{0}\right) \cdot \gamma_{f}\left(T_{\min }\right)\right] d x,
\end{aligned}
$$

where $x_{0}$ is the coordinate of the cooling source at the initial instant of time; $t$ is the time; $T(x, t)$ is the temperature of the wire sample at the point with coordinate $x$ at the instant of time $t ; v$ is the cooling zone movement velocity; $T^{0}$ is the temperature of the TiNi wire sample in unperturbed state; $S_{f}$ and $S_{r}$ are thermo-EMF coefficients for forward and reverse transformations; $\gamma_{f}$ is the ratio of martensite in the forward phase transformation. Equation (1) takes into account that only the part that went through a forward phase transformation on cooling undergoes a phase transformation in the reverse transition region.

Analyzing the physical model it was assumed that the sharp increase of the thermo-kinetic EMF value when moving the cooling zone through the local deformed zone is associated with the difference of potentials at the deformation zone boundaries as a result of the temperature change and differences between the thermo-EMF coefficient values on the plastically deformed (due to the accumulated defects) and undeformed zones of the TiNi wire sample. Therefore, plastic deformation of the TiNi sample is supposed to be expressed as the change of the thermo-EMF coefficient in the deformation zone, and the greater the value of the plastic deformation of the TiNi alloy is, the more the thermo-EMF coefficient changes.

To calculate the change of the thermo-kinetic EMF value when moving the cooling zone through the deformation zone a model according to which a difference of potentials associated with the difference of thermo-EMF coefficients and temperature occurs at the right and left boundaries of the deformation zone (Fig. 1) was suggested.

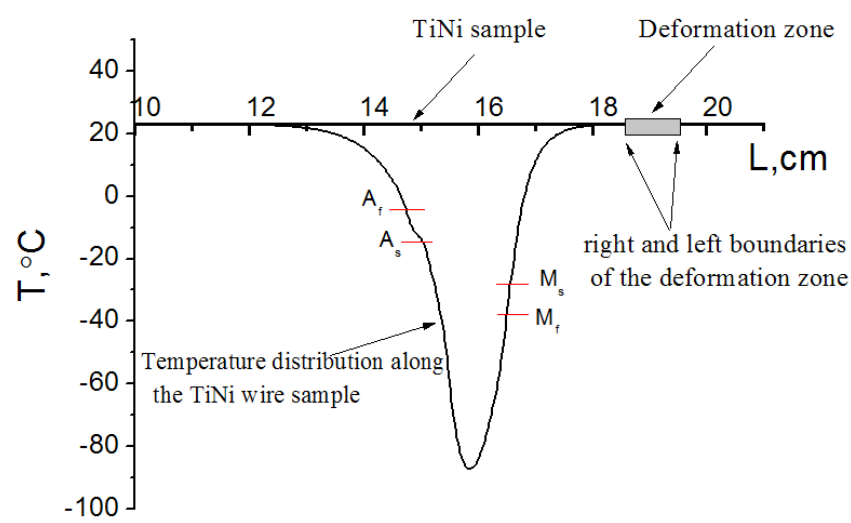

Fig. 1. Temperature distribution along the TiNi wire sample with the deformation zone.
To calculate the EMF $\left(E^{\prime}\right)$ behavior when moving the cooling zone through the local deformation zone of the TiNi sample the following formula was suggested:

$$
\begin{aligned}
E^{\prime}\left(S_{2}\right) & =\int_{t_{0}}^{t}\left[\left(S_{1}\left(T\left(x_{L}, t\right)\right)-S_{2}\right) \cdot T\left(x_{L}, t\right)-\right. \\
& \left.-\left(S_{1}\left(T\left(x_{R}, t\right)\right)-S_{2}\right) \cdot T\left(x_{R}, t\right)\right],
\end{aligned}
$$

where $x_{L}$ is the coordinate of the deformation zone left boundary, $x_{R}$ is the coordinate of the deformation zone right boundary, $S_{1}\left(T\left(x_{L}, t\right)\right)$ is the thermo-EMF coefficient of the TiNi wire sample at a given point, $S_{2}$ is the thermo-EMF coefficient in the deformation zone of the wire sample. It should be noted that $S_{1}\left(T\left(x_{L}, t\right)\right)$ is a function of temperature and changes significantly in the regions of phase transitions, $S_{2}$ is considered to be constant in the regions of phase transitions.

To calculate the temperature distribution along the sample when moving the cooling zone the case is considered when:

- at the cooling source location, cooling of the wire sample to temperatures lower than $M_{f}$ is done instantly;

- the velocity of the cooling source movement along the wire sample is constant;

- the heat energy transfer along the length of the sample on either side of the cooling zone occurs due to heat conductivity;

- a convective heat exchange of the sample with the environment takes place.

In view of the assumptions made the temperature distribution along the sample is expressed by the inhomogeneous second order partial differential equation:

$$
\rho^{\prime} c \frac{\partial T}{\partial t}=\frac{\partial}{\partial x}\left(\lambda \frac{\partial T}{\partial x}\right)-Q\left(x+v_{0} t\right)-b\left(T-T^{w}\right)
$$

with initial and boundary conditions:

$$
\begin{array}{cc}
T(x, 0)=T_{0}, & 0 \leq x \leq x_{f}, \\
T(0, t)=T\left(x_{f}, T\right)=T_{0}, & t>0 .
\end{array}
$$

Here $x$ is the current coordinate of the sample, $x_{f}$ is the coordinate of the wire sample end, $c, \rho^{\prime}$ are specific heat capacity and density of the material, respectively, $\lambda$ is heat conductivity, $b=p a / S, p, S$ are the perimeter and cross sectional area of the wire, $a$ is the coefficient of heat exchange with the environment, $T^{w}$ is the environment temperature, $V_{0}$ is the cooling zone movement velocity, $Q$ is the intensity of the internal heat absorption source.

Since the process of the conductor cooling is accompanied by nucleation of crystals of a new phase, it results in the change of physical properties of the TiNi alloy. In particular, specific heat capacity as a part of equation (3) is a function of temperature. To determine its change the following dependence was used [16]:

$$
C(T)=C_{0}+C_{1}^{\prime}\left[\sin \left(2 \pi \frac{T-A_{s}}{A_{f}-A_{s}}-\frac{\pi}{2}\right)+1\right],
$$

where $C_{1}^{\prime}$ is the material constant. When calculating the temperature distribution along the wire sample (Fig. 2) the following parameters were set: characteristic temperatures of martensitic transformations $M_{s}=-27^{\circ} \mathrm{C}, M_{f}=-37^{\circ} \mathrm{C}$, $A_{s}=-15^{\circ} \mathrm{C}, A_{f}=-4^{\circ} \mathrm{C}$; the cooling source velocity is $0.4 \mathrm{~cm} / \mathrm{s}$, the cooling source length is $1 \mathrm{~cm}$. 
As is seen in Fig. 2, the temperature distribution near the moving cooling zone is established by the 5th second; after that temperature distribution remains constant in time and only changes its location. Due to the change in heat capacity, a peak of reduction in temperature is observed (Fig. 2b) in the region of the reverse phase transformation, while in the region of the forward phase transformation the change in heat capacity does not contribute significantly to the temperature change.

To find the value of the thermo-EMF coefficient in heterophase systems, the following formula can be used [17]:

$$
S(T)=\frac{\left(\rho(T) \lambda(T)-\rho_{2} \lambda_{2}\right) \cdot\left(S_{1}-S_{2}\right)+S_{2} \cdot\left(\rho_{1} \lambda_{1}-\rho_{2} \lambda_{2}\right)}{\rho_{1} \lambda_{1}-\rho_{2} \lambda_{2}} .
$$

In the present model the generalized electrical resistivity $\rho$ and heat conductivity $\lambda$ can be considered as normalized sums of phase contributions:

$$
\rho=\frac{\sum \gamma_{i}(T) \rho_{i} g_{i}(\rho)}{\sum \gamma_{i}(T) g_{i}(\rho)}, \quad \lambda=\frac{\sum \gamma_{i}(T) \lambda_{i} g_{i}(\lambda)}{\sum \gamma_{i}(T) g_{i}(\lambda)},
$$

where the sum of phase concentrations $\gamma_{i}$ is 1 , and configuration parameters $g_{i}(\rho)$ and $g_{i}(\lambda)$ along electric (thermal) currents are as follows:

$$
g_{i}(\rho)=\frac{3 \rho}{A \rho+(3-A) \cdot \rho_{i}}, \quad g_{i}(\lambda)=\frac{3 \lambda}{A \lambda+(3-A) \cdot \lambda_{i}} .
$$

Here, parameter $A$ is equal to 0 for the parallel connection of phases, and to 3 for the case of successively connection. Intermediate values of $A(0<A<3)$ correspond to interpolated configuration of phases in definite directions (e.g. prolate or oblate ellipsoids) $[18,19]$. To describe the dependences of internal variable $\gamma_{i}$ on temperature $T$ at forward $\gamma_{f}$ and reverse $\gamma_{r}$ for martensite transformations, Liang-Rogers function [20] is used:

$$
\begin{array}{cc}
\gamma_{f}=\frac{1}{2}\left[\cos \left(a_{M}\left(T-M_{f}\right)\right)+1\right], \quad M_{f}<T<M_{s}, \\
\gamma_{r}=\frac{1}{2}\left[\cos \left(a_{A}\left(T-A_{s}\right)\right)+1\right], \quad A_{s}<T<A_{f},
\end{array}
$$

where values $a_{A}$ and $a_{M}$ have following values: $a_{M}=\pi /\left(M_{s}-M_{f}\right)$, $a_{A}=\pi /\left(A_{f}-A_{s}\right)$. To calculate the change in EMF value when moving the cooling zone through the deformation zone the following values were set: $S_{a}=8 \mathrm{mV} / \mathrm{K}, S_{m}=11 \mathrm{mV} / \mathrm{K}$
(thermo-EMF coefficients for austenite and martensite, respectively) [21], the length of the deformation zone is $0.18 \mathrm{~cm}$.

Two successive EMF peaks opposite in sign can be seen on the curve (Fig. 3) calculated according to equations (2) - (7) in the process of movement of the cooling zone along the local deformation zone with the velocity of $0.4 \mathrm{~cm} / \mathrm{s}$. The first peak occurs when moving the zone with the forward phase transformation, which is close to the cooling zone (Fig. 1), through the deformation zone; the second one can be observed when moving the zone with the reverse phase transformation through the deformation zone. The theoretical results obtained are in good agreement with experimental studies [13].

It can be further assumed that the length of the deformation zone has an impact on the value of the thermo-kinetic EMF when moving the cooling zone through the deformation zone, since its elongation results in the increase in the temperature difference on its boundaries, which, consequently, affects the potential difference on the boundaries and the change in the thermo-kinetic EMF value. The change in EMF when moving the cooling zone through the deformation zone of the TiNi wire samples of different lengths was calculated according to formulas (2) - (7).

Indeed, according to the results obtained by calculation (Fig. 4), the change in the thermo-kinetic EMF value increases with the increase in the length of the deformation zone up to $1.3 \mathrm{~cm}$, while with further elongation the EMF change remains constant and two EMF peaks equal in value and opposite in sign appear. The peaks can be explained by a successive movement of the cooling zone through the deformation zone boundaries (Fig. 5).

Therefore, as long as the length of the deformation zone is less than that of the cooling zone $\left(L_{\text {def. }}<1.3 \mathrm{~cm}\right)$, the peaks overlap each other and their effective value is less than the value of each peak. As soon as the deformation zone length exceeds that of the cooling zone, the peaks no more overlap and their values do not change. In the present paper we consider the case when the velocity of the cooling zone movement along the conductor is constant and, consequently, the length of the cooling zone does not change, which results in the thermo-kinetic EMF value only depending on the change in the deformation zone length.

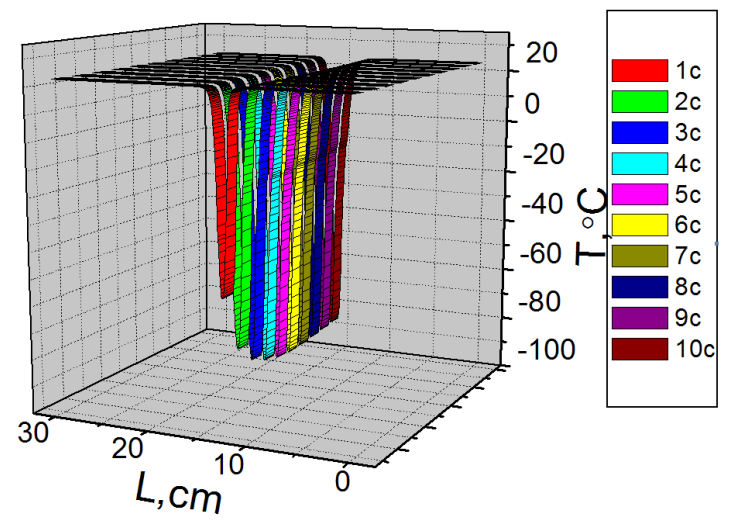

a

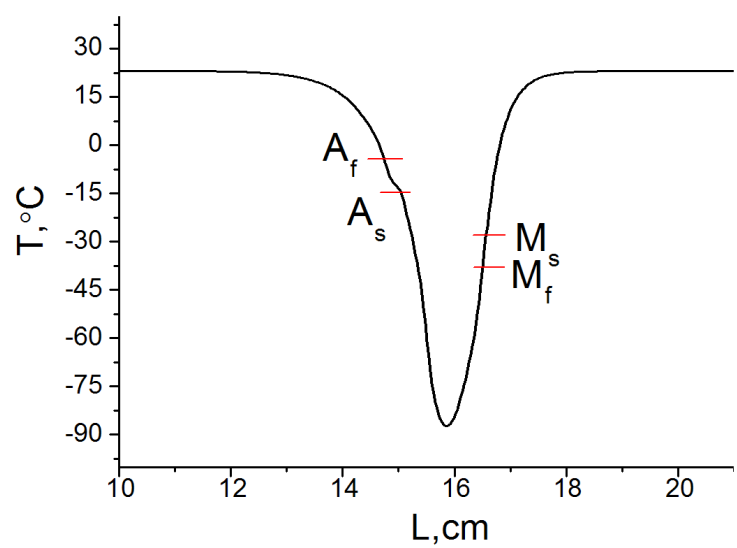

b

Fig. 2. Temperature distribution of the TiNi wire sample near the cooling zone: per the first ten seconds (a); per the tenth second (b). 
The same change of the thermo-kinetic EMF can occur if the cooling zone length changes due to the change in the movement velocity of the cooling zone while the length of the deformation zone remains constant. Therefore, the change in the thermo-kinetic EMF value when moving the cooling zone through the deformation zone depends on the cooling zone length to the deformation zone length ratio.

Basing on the model suggested the maximum change in the thermo-kinetic EMF (in the case when the length of

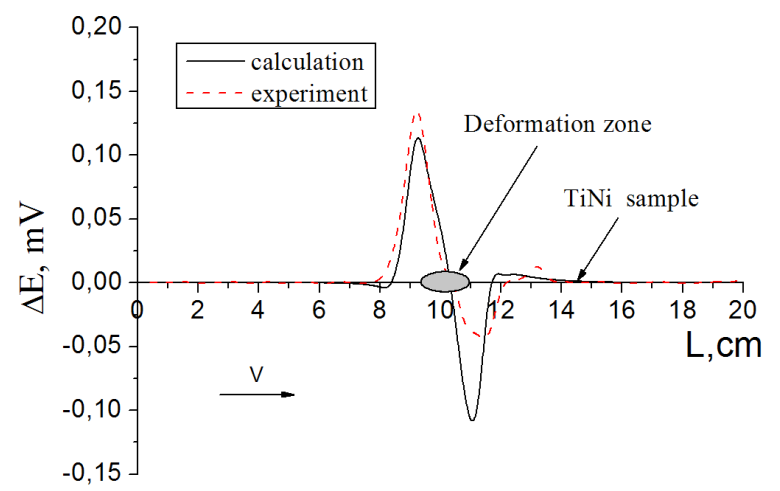

Fig. 3. Change in thermo-kinetic EMF value when moving the cooling zone through the deformation zone.

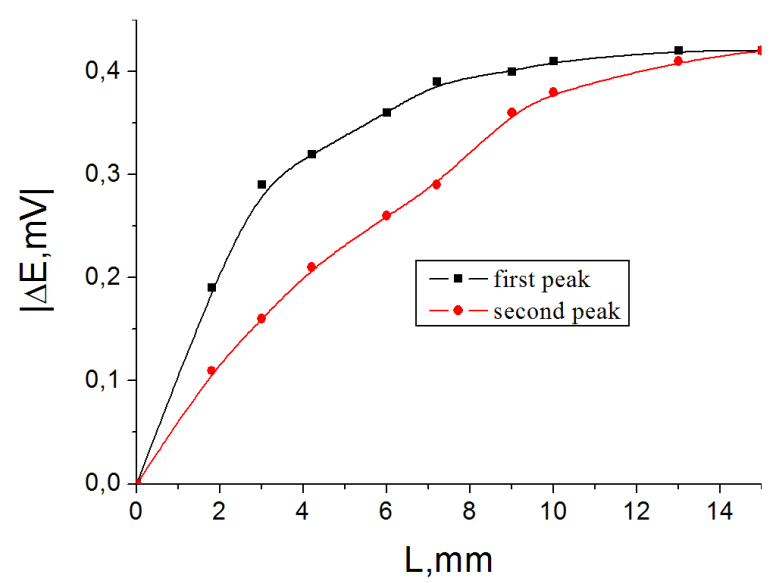

Fig. 4. The calculated dependence of the change in the thermokinetic EMF value on the length of the deformation zone.

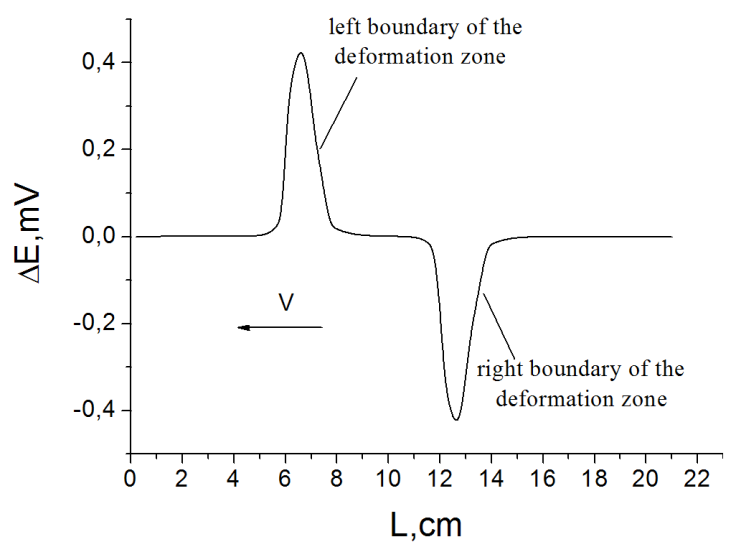

Fig. 5. The calculated dependence of the change in the thermo-kinetic EMF value during the movement of the cooling zone through the 6 $\mathrm{cm}$ long deformation zone. the deformation zone exceeds that of the cooling zone) is observed when moving the region of minimal temperature in the cooling zone through the boundaries of the deformation zone. Therefore, according to the physical model suggested the change in the thermo-kinetic EMF value when moving the cooling zone through the deformation zone depends on the cooling zone length to the deformation zone length ratio as well as the value of minimal temperature in the cooling zone. The assumptions call for further experimental studies for confirmation.

\section{Conclusions}

In the present paper, expressions to calculate the change in the thermo-kinetic EMF value when moving the cooling zone through the deformation zone in TiNi alloy have been obtained. It has been established that two successive EMF peaks of opposite signs are observed when moving the cooling zone through the deformation zone $\left(L_{\text {def. }}<1.4 \mathrm{~cm}\right)$. The first peak occurs when moving the zone with the forward phase transformation, which is close to the cooling zone, through the deformation zone; consequently, the second one can be observed when moving the zone with the reverse phase transformation through the deformation zone. The change in the thermo-kinetic EMF value when moving the cooling zone through the deformation zone can be explained by the potential difference on the boundaries of the deformation zone due to the temperature change and the difference in the thermo-EMF coefficient value in the plastically deformed and undeformed zones of the TiNi wire sample. The calculation results of the physical and mathematical model used to estimate the change in the thermo-kinetic EMF value when moving the cooling zone through the deformation zone are in good agreement with experimental data.

Basing on the analysis of the results obtained it was assumed that the change in the thermo-kinetic EMF value when moving the cooling zone through the deformation zone depends on the value of the minimal temperature in the cooling zone as well as on the ration of cooling zone length to the deformation zone one. Direct studies of the impact of the deformation zone length on the thermo-kinetic EMF value at fixed velocity of the cooling zone movement and, thereby, its constant length, have been carried out. It has been deduced from theoretical studies that the value of the maximum change in the thermo-kinetic EMF when moving the cooling zone through the deformation zone increases with the increase of the deformation zone length up to $1.3 \mathrm{~cm}$, while with further elongation the EMF change remains constant and there appear two EMF peaks equal in value and opposite in sign and corresponding to the left and right boundaries of the deformation zone. Therefore, as long as the length of the deformation zone is shorter than that of the cooling zone $\left(L_{d e f}<1.3 \mathrm{~cm}\right)$, the peaks overlap each other and their effective value is less than the value of each peak. As soon as the deformation zone length exceeds the one of the cooling zone, the peaks no more overlap and their heights do not change. The results obtained call for further experimental studies to confirm the impact of the cooling zone length to the deformation zone length ratio on the change in the 
thermo-kinetic EMF value. The results of calculation of the change in the thermo-kinetic EMF when moving the cooling zone through the deformation zone in TiNi alloys are in good agreement with the experimental data and can be used to develop methods and tools for homogeneity control of extended TiNi products.

\section{References}

1. K. Ootsuka, K. Sumidzu, Y. U. Sudzuki. Alloys with shape memory effect. Moscow, Metallurgy (1990) 224 p. (in Russian)

2. Z. G. Wei, R. Sandström, S. Miyazaki. Journal of Materials Science. 33, 3743 (1998). DOI: 10.1023/A:1004674630156

3. D. Mantovani. The Journal of The Minerals, Metals. 52(10), 36 (2000). DOI: 10.1007/s11837-000-0082-4

4. A.A. Luhvich, A.S. Karolik, V.I. Sharando. Structural dependence of thermoelectric properties and nondestructive testing. Minsk, Science and Technology (1990) 192 p. (in Russian)

5. Z.S. Basinski, J. S. Dugdale, A. Howie. Philos. Mag. 8(96), 1989 (1963).

6. A.S. Karolik. In: Computer methods and inverse problems in nondestructive testing and diagnostics. Minsk (1995) $210 \mathrm{p}$.

7. E. F. Furmakov. Fundamental problems of natural science. 1(21), 377 (1999). (in Russian)

8. E. F. Furmakov. In: Krym 2004. Sudak (2004) 11 p. (in Russian)

9. V. V. Rubanik, V. V. Rubanik Jr., O. A. Petrova-Burkina. In: ESOMAT. S.-Pb. (2012) 40 p.

10. V. V. Rubanik, V. V. Rubanik Jr., O. A. Petrova-Burkina. In: The influence of electromagnetic fields on the plasticity and strength of materials. Novokuznetsk (2011) 60 p. (in Russian)

11. V. V. Rubanik, V. V. Rubanik Jr., O. A. Petrova-Burkina. In: International Conference on Martensitic Transformation (ICOMAT 2011). Japan (2011) 180 p.

12. V. V. Rubanik, V. V. Rubanik Jr., A. V. Lesota. In: SMA: properties, technologies, prospects. Vitebsk, VGTU (2014) 33 p. (in Russian)

13. V. V. Rubanik, V.V. Rubanik Jr., A. V. Lesota. In: Physical material: VII of the International School with elements of scientific school for young people. Tolyatti (2016) 273 p. (in Russian)

14. V.V. Rubanik, A.V. Lesota, V.V. Rubanik Jr. Letters on materials. 7(2), 96 (2017). (in Russian) DOI: $10.22226 / 2410-3535-2017-2-96-100$

15. S. E. Kulkova, D. V. Lalujsky, I. Y. Smolin. Phys. Solid State. 43(4), 737 (2001). (in Russian)

16. Y. N. Vyunenko. In: Advanced technologies and methods of control. Vitebsk (2009) 384 p. (in Russian)

17. V. V. Shchennikov, S. V. Ovsyannikov, G. V. Vorontsov. Physica Status Solidi B. 241(14), 3203 (2004). DOI: $10.1002 /$ pssb.200405245

18. S. V. Ovsyannikov, V. V. Shchennikov, I. A. Komarovskii. Proceedings of the SPIE. 7978, (2011). DOI: $10.1117 / 12.881913$

19. V.V. Shchennikov, S. V. Ovsyannikov, A.Y. Derevskov, V.V. Shchennikov Jr. Journal of Physics and Chemistry of Solids. 67(9), $2203 \quad$ (2006). DOI: $10.1016 /$ j.jpcs.2006.06.004

20. C. Liang, C. Rogers. Journal of Intelligent Material System and Structure. 1(2), 207 (1990). DOI: $10.1177 / 1045389 X 9000100205$

21. J.E. Hanlon, S.R. Butler, R.J. Wasilewski. Trans. Met. Soc. AIME. 239, 1323 (1967). 